\title{
Retinal Vascular Tree Reconstruction With Anatomical Realism
}

\author{
Kai-Shun Lin, Chia-Ling Tsai*, Member, IEEE, Chih-Hsiangng Tsai, Michal Sofka, Shih-Jen Chen, \\ and Wei-Yang Lin, Member, IEEE
}

\begin{abstract}
Motivated by the goals of automatically extracting vessel segments and constructing retinal vascular trees with anatomical realism, this paper presents and analyses an algorithm that combines vessel segmentation and grouping of the extracted vessel segments. The proposed method aims to restore the topology of the vascular trees with anatomical realism for clinical studies and diagnosis of retinal vascular diseases, which manifest abnormalities in either venous and/or arterial vascular systems. Vessel segments are grouped using extended Kalman filter which takes into account continuities in curvature, width, and intensity changes at the bifurcation or crossover point. At a junction, the proposed method applies the minimum-cost matching algorithm to resolve the conflict in grouping due to error in tracing. The system was trained with 20 images from the DRIVE dataset, and tested using the remaining 20 images. The dataset contained a mixture of normal and pathological images. In addition, six pathological fluorescein angiogram sequences were also included in this study. The results were compared against the groundtruth images provided by a physician, achieving average success rates of $88.79 \%$ and $90.09 \%$, respectively.
\end{abstract}

Index Terms-Kalman filter, retinal vascular tree, vascular tree reconstruction.

\section{INTRODUCTION}

I DENTIFYING the organization of vascular trees provides valuable information for physicians performing surgical planning and outcome assessment, and for monitoring the progression of vascular diseases [1]. For treatment of strokes, with the complete map of the vascular trees, neurosurgeons, and radiologists can make the best decision about vessel occlusion and ensure collateral flow provided by other parts of the circulation system [2]. A liver transplant is a procedure in which a healthy voluntary donor gives a part of his or her liver to another person.

Manuscript received November 30, 2011; revised April 3, 2012 and June 22, 2012; accepted August 10, 2012. Date of publication August 23, 2012; date of current version November 22, 2012. This work was supported by the National Science Council, Taiwan under Grant NSC98-2221-E-194-039-MY3. Asterisk indicates corresponding author.

K.-S. Lin, C.-H. Tsai, and W.-Y. Lin are with the Department of Computer Science and Information Engineering, National Chung Cheng University, Chiayi, Taiwan (e-mail: kasuekimo@yahoo.com.tw; ctsai@iona.edu; wylin@cs.ccu.edu.tw).

${ }^{*}$ C.-L. Tsai is with the Department of Computer Science, Iona College, New Rochelle, NY 10801 USA (e-mail:ctsai@iona.edu).

M. Sofka is with the Siemens Corporate Research, Princeton, NJ 08540 USA (e-mail: sofkam@rpi.edu).

S.-J. Chen is with the Taipei Veterans General Hospital, National Yang-ming University, Taipei 112, Taiwan (e-mail: sjchen96@gmail.com).

Color versions of one or more of the figures in this paper are available online at http://ieeexplore.ieee.org.

Digital Object Identifier 10.1109/TBME.2012.2215034

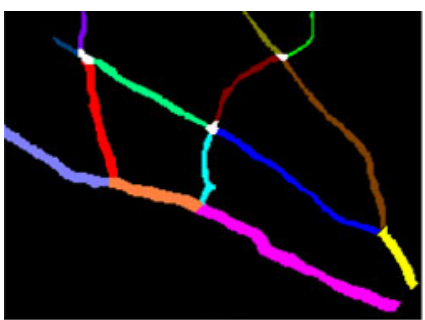

(a)

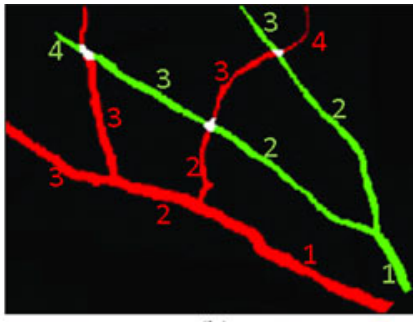

(b)
Fig. 1. Retinal vascular tree reconstruction with anatomical realism. (a) Ungrouped vessel segments, each assigned a unique color. (b) The expected result of vessel tree reconstruction with anatomical realism. Segments of the same tree are connected and given the same color-each segment is connected to its child segment(s) with a number that immediately follows its number.

A careful analysis of the branching pattern and morphology of the vasculature can reveal the suitability of the donor. For oncology resection on a patient with liver cancer or liver metastasis, identifying vascular territories is crucial to providing resection proposals and for preoperatively estimating resection volumes and patient outcomes [3].

The goal of the proposed system is to reconstruct topologies of the retinal vascular trees with anatomical realism. In retinal images, vessels are organized in trees with roots inside the optic disk. Following the main arteries and veins, vessels branch out until capillaries (the thinnest retinal vessels) are reached. To reconstruct the vascular trees, segments from vessel tracing should be connected to the parent segments based on physiological properties, instead of proximity. In Fig. 1(a), the vessel segments, each assigned a unique color, can be the result of running a vessel tracing algorithm. If the vessels are connected (grouped) with anatomical realism, the expected result is shown in Fig. 1(b), where each segment is connected to its child segment(s) with the same color and with a number that immediately follows its number. Extracting the trees allows the visualization of the vasculature, which can facilitate studies of blood flow by displaying traversal from any given point to another within the same tree. It is also a precursor for automated quantification of geometrical and topological properties of veins and arteries for studies of medical conditions such as hypertension [4].

This proposed method performs grouping on the extracted vessel segments at junction points by considering the continuity of vessel properties. Challenges are as follows.

1) Incomplete tracing: Vessels of low contrast are often partially traced, especially near junctions with other vessels.

2) Unreliable tracing at junctions: Certain vessel properties, such as vessel width and vessel direction, are less reliable near and at junctions due to tracing limitations. In addition, 
vessels crossing at a very sharp angle sometimes can be incorrectly traced as one big vessel at the junction. As a result, a crossover is turned into two bifurcations (see Fig. 7).

To improve the tracing of low contrast vessels based on [5], both vesselness and connectedness are exploited to maximize the completeness of vessel extraction. For grouping with unreliable trace results at the junction points, the Kalman filter and minimum-cost matching are combined to ensure the continuity of vessel segments at junction points by taking into account the variations in width, curvature, and intensity along vessel segments. The proposed method is an extended version of our previous method [6], [7]. Preliminary results using both the tracing and grouping with only the Kalman filter can be found in our earlier publications [6], [7]. Contributions of this study include the enhancement using minimum-cost matching for higher robustness, complete analysis of the system using the DRIVE dataset, and the generalization of the algorithm for both color and fluorescein images. In this paper, we include the discussion of all major components of the system for completeness.

\section{RELATED WORK}

Automated analysis of retinal images has been an active research topic. There is a strong medical need of measurements specific to vascular trees [4], [8]-[14]. One application is the measurement of the the arteriolar-to-venous (A/V) diameter ratio [4], [8]-[11]. Clinical studies suggest that narrowing of the arterial blood vessels in the retina may be an early indicator of hypertension [11]-[14] and atherosclerosis, which is a process of inflammation in the vessel wall that leads to plaque formation and a reduction in arterial flexibility [15]. Vascular trees can also be used to analyze important features in retinal images, such as the optic disk and macula. This is crucial for diagnosing diseases such as macula degeneration-drusens close to the macula need immediate attention, whereas drusens on the periphery might need to be monitored only.

Two major approaches on the construction of vascular structure appear in the literature. One approach, named growingbased method, is to grow either the vascular regions, which are later transformed into vascular trees [3], [16], or the skeletons directly [17], [18], starting from some seed points. The other approach, named the grouping-based method, is to build the trees from detected vessel segments using perceptual grouping techniques [19]-[25].

For growing-based methods, Selle et al. [3] segmented vessels using a threshold-based region-growing method, with the threshold value automatically generated. The segmented vascular structure is skeletonized, and transformed into a vascular "forest" that contains a number of directed acyclic graphs, using a graph theoretical method. Yim et al. introduced a similar approach. [16]. The tree is generated by the ordered region growing (ORG) technique. A seed is provided at the root of the tree. The growth, started from a user-defined point, is in the direction of higher intensity. The result of ORG is a graph. However, it is not clear how the ramifications are handled. Following ORG is either skeletonization or pruning. Instead of extracting the vas- cular regions, Haris et al. [17] and Liu and Sun [18] traced the vessel centerlines. In [17] and [18], the tree is constructed as the vessels are being traced. Starting from a seed point, vessel pixels are found recursively along the vessel centerline. Ramifications are handled in a breadth-first-search manner.

For grouping-based methods, Jomier et al. [19] and Bullitt et al. [20] both performed grouping of vessel segments to form a minimum spanning tree, but with different minimization criteria. The authors in [19] minimized the Mahalanobis distance of features defined at each junction, while [20] performed the minimization based on linear distance and image intensity in suspected regions of connection. Al-Diri et al. [21] presented self-organizing feature maps to model cost functions for the junctions in order to resolve the configuration of local sets of segment ends, thus determining the network connectivity. Montesinos et al. [22] constructed trees by performing local to the global optimization of an active contour by dynamic programming. Coppini et al. [23] proposed a bottom-up coronary artery extraction method based on angiogram edges. These edges are grouped into edge segments, which are organized to bar primitives, and ultimately linked to form artery segments. An algorithm developed by Deschamps and Cohen [24], [25] first converts the vascular image to a threshold "vessel potential" image and performs perceptual grouping and contour completion. This fast marching algorithm identifies contours as minimal paths between connected components.

\section{VESSEL SEGMENT EXTRACTION}

In order to identify vessel pixels, we propose an enhanced method that improves upon an existing vessel extraction algorithm [6]. This method combines matched filter responses, confidence measures, and vessel boundary measures. Matched filter responses are derived in scale space to extract vessels of widely varying widths. A vessel confidence measure is defined as a projection of a vector formed from a normalized pixel neighborhood onto a normalized ideal vessel profile. Vessel boundary measures and associated confidences are computed at potential vessel boundaries. Combineding these responses forms a 6-D measurement vector at each pixel. A learning technique then maps this vector to a likelihood ratio that measures the likelihood ratio vesselness (LRV) at each pixel. The learning technique is the optimal test statistic by the Neyman-Pearson Lemma [26]. The result of LRV computation for Fig. 2(a) is Fig. 2(b), which is enhanced for display. Tracing of vessel segments consists following three major steps.

1) Seed point initialization: The algorithm analyzes the image along a coarse grid to detect seed locations on blood vessels which are gray-scale minima between opposite signed, close-to-parallel 1-D edges. The direction of tracing at a seed point is the eigenvector corresponding to the smallest eigenvalue of the local, multiscale Hessian around the seed location. Vessel width is found from the maximum matched filter response in scale space applied in this direction. The seed points are sorted based on strength so that when tracing starts, the most reliable points are selected first. 


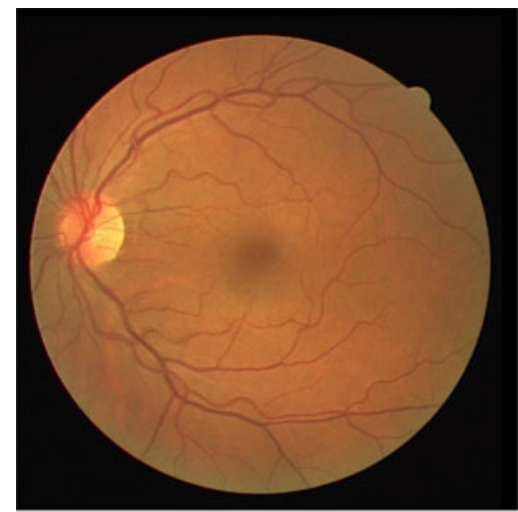

(a)

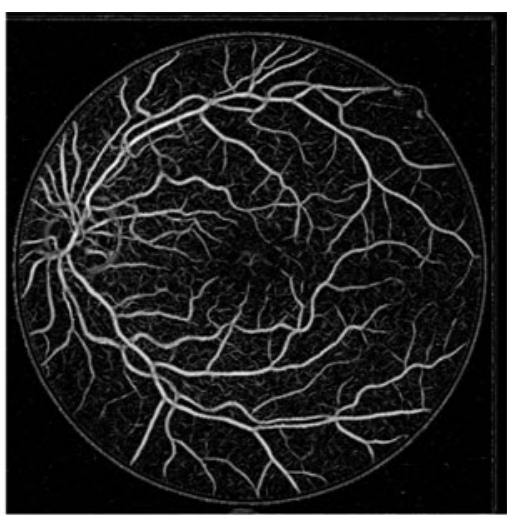

(b)

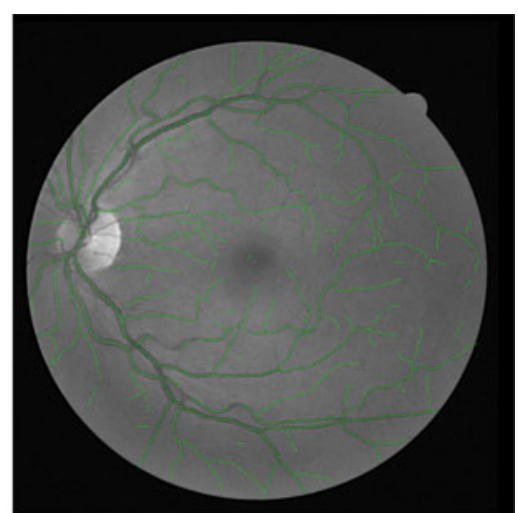

(c)

Fig. 2. Example shows the results of LRV and LRV tracing. (a) Color retinal image. (b) LRV result enhanced for display. (c) Result of LRV tracing.

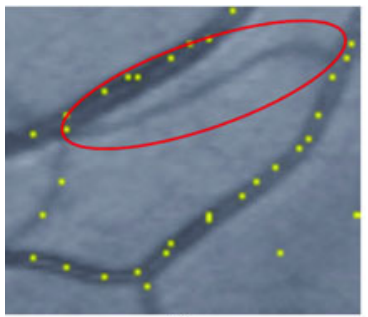

(a)

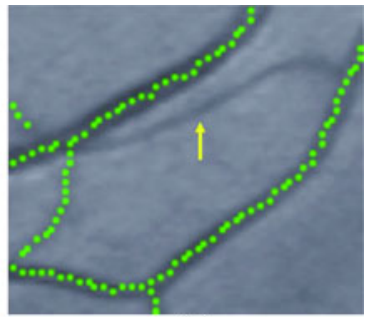

(b)
Fig. 3. Failure in tracing due to missing seed points. (a) Yellow dots are the seed points. The red ellipse contains the vessel without seeds. (b) Result of tracing. The arrow indicates the missing trace.

2) Recursive tracing: Starting from a seed point that is not on any previously traced vessel segments, the vessel location, vessel tangent direction, and vessel scale are updated at each step of tracing. This is achieved by searching for the maximum scale-space response over \pm 20 degrees change in orientation, \pm 1 steps in scale space, and \pm 2 pixel steps in the normal direction. If the largest response is nonpositive, tracing is terminated. Otherwise, a step of two pixels is taken along the updated tangent direction, and the process is repeated.

3) Pruning: Recursive tracing using only matched filter often results in overtraced results. After all seed points have been visited, the postprocessing verification step removes trace points with the LRV value below a threshold set to 2 as in [5]. Here, we adopt the threshold value for vesselness directly from the original paper on LRV [5]. The range of this vesselness measure is from 0 to $10^{29}$. In particular, the LRV measure of 1.0 indicates an equal chance that a pixel belongs to vessel or nonvessel. When the value of LRV measure is 2.0, the current pixel is twice as likely to be on-vessel as off-vessel. Consequently, by setting the threshold to 2 , we are more confident that a vascular structure is present. Fig. 2(c) shows the tracing results.

\section{A. Improving Seed Points Detection}

Potential problems with LRV tracing include missing seed points for small vessels, as Fig. 3 shows, and determination of the threshold value in pruning. To mitigate both problems, the proposed LRV tracing method includes the detection of junction points for the placement of extra seed points and combination of vesselness and connectedness for pruning to alleviate the dependence of a single threshold value on LRV. These enhancements are based on tensor voting [27] to determine whether a pixel is likely to be on a junction or a curve.

When tensor voting is performed, each pixel encoded as a plate tensor sends its tensor to its neighboring pixels through its voting field. The result of voting at each pixel is a variancecovariance matrix consisting of eigenvalues and eigenvectors. Let $\lambda_{\min }$ and $\lambda_{\max }$ be the minimum and maximum eigenvalues, respectively. A pixel is on a curve structure if its $\lambda_{\max }$ is substantially larger than $\lambda_{\min }$, whereas a pixel may be on a junction of two or more curves if both eigenvalues are similar. In other words, $\lambda_{\max }$ provides a good indication that a pixel is on a continuous structure, such as a curve, and serves as a measure of connectedness of the pixel with its neighboring pixels. Alternately, $\lambda_{\min }$ provides the saliency for junctions. Both connectedness and junction saliency maps are obtained from the LRV image, instead of the original image. This is because the former excludes nonvessel structures with well-defined boundaries, such as the optic disk.

To maximize the likelihood that at least one seed point is found on every vessel, the proposed method includes the seed points defined by the LRV tracer [Fig. 3(a)] and the points near vessel junctions. The motivation to adopt the latter is rather intuitive, since blood vessels are naturally connected. Thus, if the parent vessel has been traced, the vessels that branch out of it are less likely to be missed if seeds are placed near the junctions. To enable identification of a junction before all vessels that come to meet are extracted, potential junction locations are detected using the junction saliency map, as Fig. 4(c) shows. The candidate junction points are obtained by performing nonmaximum suppression [28] on the junction saliency map. The circles in Fig. 4(c) indicate candidate junction points found on the junction saliency map. Given a seed point located on the center of a vessel junction, it will inevitably favor tracing along main vessel. Thus, main vessel segment will trace the junction seed and leave no seed point for branch vessel. In order to avoid such undesirable situations, we propose to place extra seed points 


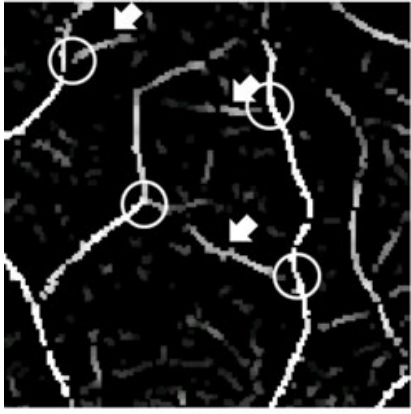

(a)

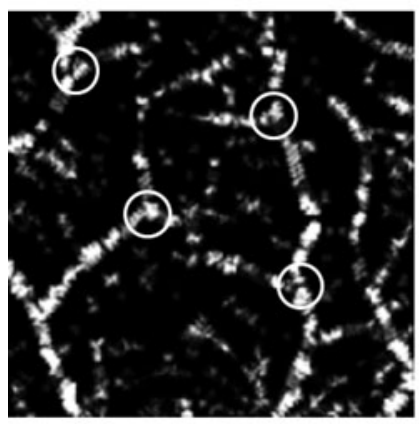

(c)

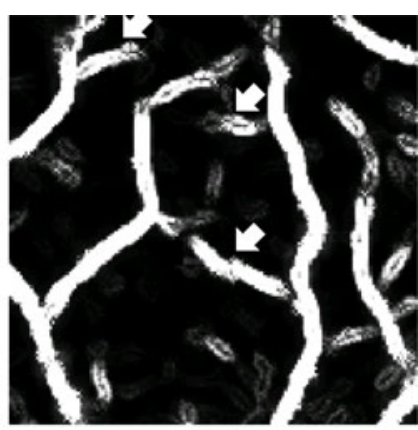

(b)

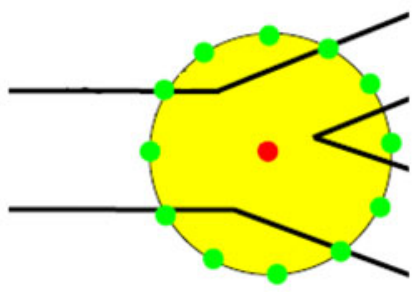

(d)
Fig. 4. Example of tensor voting on an LRV image. (a) LRV image. (b) Curve saliency map for the computation of the connectedness values. (c) Junction saliency map for computation of the extra seed points near vessel junctions. (d) Seed points near a vessel junction. The red dot indicates the center of the junction. Twelve equally spaced green dots are the seed points on the periphery of the disk centering at the junction with the radius as a function of the vessel width. In (a) and (b), the arrows indicate thin vessels enhanced in the curve saliency map. In (a) and (c), the circles are centered at the candidate junction points, which define extra seed points to be included in vessel tracing.

around a candidate junction point. At each candidate junction point, we draw a circle with radius equal to the vessel width extended by 5 pixels. On the periphery of this circle, we generate 12 equally spaced points, as shown in Fig. 4(d), and utilize them as the extra seed points.

\section{B. Robustness of Pruning}

A tradeoff must be made between false positive and false negative rates if pruning of the overtraced result uses only a single threshold value on the LRV. Reducing the threshold value increases both the true and false positives. To improve the robustness of pruning, the proposed method considers both vesselness and connectedness measures of a pixel-points with high connectedness are likely to be on a continuous structure, such as a vessel, even if the vesselness measure falls below the threshold value. The connectedness value for each pixel is obtained directly from the curve saliency map, as shown in Fig. 4(b). We obtain a connectedness threshold using a simple global thresholding method to partition the curve saliency map histogram $H$. However, since the maximum saliency value can go as high as $5 \times 10^{4}$, pixels with high saliency values are iteratively eliminated to reduce their effect on the computation of the threshold value. The algorithm is as follows, starting with $n$ being the maximum saliency value.
1) Calculate the average curve saliency value $T=$ $\frac{\sum_{i=1}^{n} i H[i]}{\sum_{i=1}^{n} H[i]}$.

2) Compute the average low curve saliency value $\mu_{1}=$ $\frac{\sum_{i=1}^{T} i \times H[i]}{\sum_{i=1}^{T} H[i]}$ and average high curve saliency value $\mu_{2}=$ $\frac{\sum_{i=T+1}^{n} i \times H[i]}{\sum_{i=T+1}^{n} H[i]}$ of the two partitions.

3) Update $n: n=\frac{\mu_{1}+\mu_{2}}{2}$.

4) Repeat steps 1 to 3 for 3 times.

5) Return $T$ as the threshold value for connectedness.

Given traces produced by the matched filter, trace points of each trace are revisited consecutively, and the vesselness and connectedness measures are examined. If the former is below the threshold, which is set to 2 , the pixel and the remaining ones in the trace, is discarded if 1) it is one of the first three trace points, 2) previous two trace points have vesselness measures below the threshold, or 3) the connectedness is below the threshold.

At a crossover, it is possible that the vessel tracer follows a vessel that is not anatomically connected to its current vessel. As a result, the extracted vessel segment may contain segments from different blood vessels when tracing terminates. Before grouping the vessel segments for vessel tree reconstruction, every vessel segment is broken up into shorter pieces at junctions with other vessel segments to ensure that the basic components for grouping are segments that contain only points from the same blood vessel.

\section{VESSEL GROUPING}

Given the set of traced vessel segments, the grouping algorithm connects segments to restore the topology of an anatomically realistic vascular tree. Fig. 5 presents an overview of the grouping process. Segments are prioritized for grouping. Initially, segments that intersect with the boundary of the optic disk (chosen manually) are assigned the first priority. Those segments are roots of constructed vessel trees. As grouping progresses, segments close to vessels that are already part of constructed trees are assigned the second priority. Segments of the same priority are handled in any order before segments of the next priority.

The process of connecting the segments to form a vessel starts from an ungrouped segment with the highest priority, and iterates three steps: 1) iteratively correcting and learning the continuity pattern of the current segment using the extended Kalman filter, 2) picking the next segment with the highest continuity in the neighborhood of the end point of the current segment, and 3) regrouping using minimum-cost matching if the chosen segment has been grouped. If the next segment cannot be located, the process terminates for the current vessel, and the priorities of the remaining ungrouped segments are updated if necessary. This tree reconstruction process terminates when no segment of first or second priority can be found. Constructed vessels that are initialized with vessels of second priority are connected to the nearest vessel and become part of a vessel tree. The final result is a set of trees whose root segments intersect with the boundary of the optic disk. 


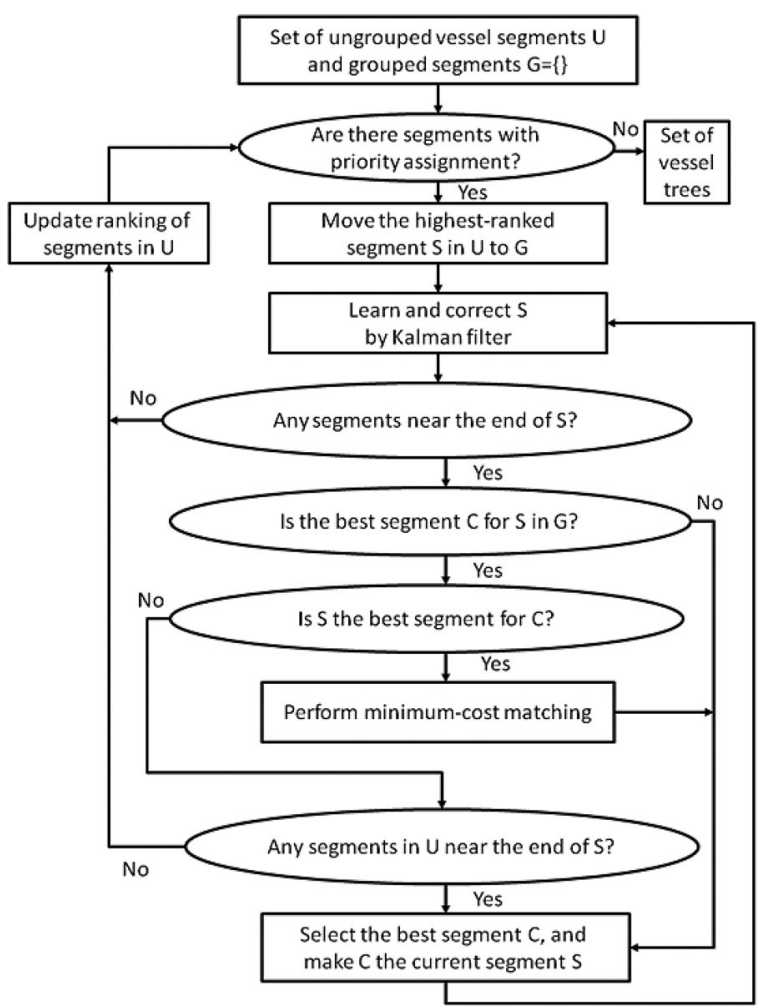

Fig. 5. Flow chart of perceptual grouping process.

\section{A. Vessel Grouping by the Kalman Filter}

Some vessel properties vary gradually during the grouping process. To take this observation into consideration, we assume constant changes of two important properties: vessel width and intensity. Using the training images from the DRIVE dataset, it is possible to calculate the average changes from one vessel point to the next coming from the direction of the root. The average decrements for vessel width and intensity are 0.0147 pixel and 0.23 gray level, respectively. In addition, the vessel direction usually varies continuously along a vessel segment. The next vessel segment can be extrapolated along the vessel direction. The proposed method adopts an extended Kalman Filter to learn the characteristics of a vessel segment to ensure best continuity when choosing for the next segment for grouping, as Fig. 6 shows. Each trace point $\mathbf{p}_{k}$ is associated with a seven-tuple, state vector $\mathbf{X}_{k}=\left(x_{k}, y_{k}, G_{k}, d_{k}, \theta_{k}, \dot{G}_{k}, \dot{d}_{k}\right)$, where $x_{k}$ and $y_{k}$ form the coordinate, $G_{k}$ is the intensity value, $d_{k}$ is the vessel width, $\theta_{k}$ is the direction of the vessel at $\mathbf{p}_{k}, \dot{G}_{k}$ is the change in the intensity value, and $\dot{d}_{k}$ is the change in the vessel width. If a color image is given as the input, $G_{k}$ is the green channel that gives the highest contrast between the vessels and the background [29].

At $\mathbf{p}_{k}$, the system model and the observation model are, respectively, defined as

$$
\begin{aligned}
& \mathbf{X}_{k}=f_{k-1}\left(\mathbf{X}_{k-1}\right)+\mathbf{W}_{k-1} \\
& \mathbf{X}_{k}^{o}=h_{k}\left(\mathbf{X}_{k}\right)+\mathbf{V}_{k}
\end{aligned}
$$

where $\mathbf{X}_{k}^{o}$ is the five-tuple observation vector $\left(x_{k}^{o}, y_{k}^{o}, G_{k}^{o}, d_{k}^{o}, \theta_{k}^{o}\right)$ at $\mathbf{p}_{k}$. In the proposed approach, $\mathbf{X}_{k}^{o}$

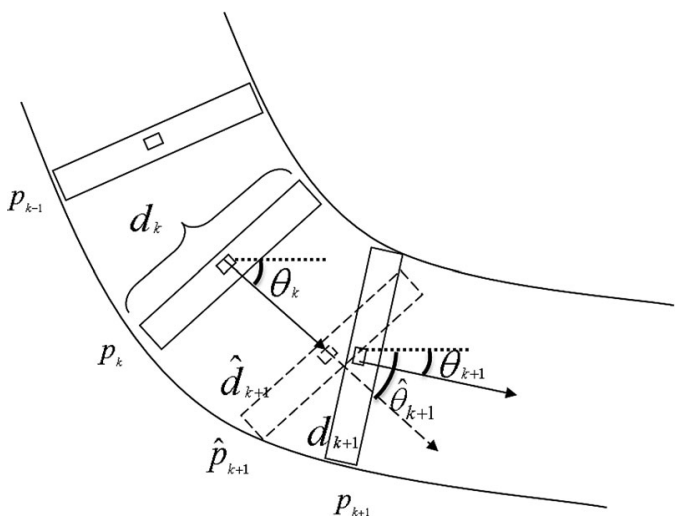

Fig. 6. Geometric illustration of the estimation-update process of grouping The vessel point position is estimated to $\hat{p}_{k+1}$ at $d_{k}$ pixels ahead and adjusted to the actual one $p_{k+1}$ by the vessel tracer. $d_{k}$ and $\theta_{k}$ are vessel width and vessel tangent direction at the $k$ th position.

comes from the LRV vessel tracer. $\left(x_{k}^{o}, y_{k}^{o}\right)$ is the location of the centerline point. $G_{k}^{o}$ is the gray-scale intensity value at $\left(x_{k}^{o}, y_{k}^{o}\right) . d_{k}^{o}$, is the width which is the distance between the two edge points detected by LRV along the line perpendicular to the vessel direction at $\left(x_{k}^{o}, y_{k}^{o}\right) . \theta_{k}^{o}$ is the angle between the $x$-axis and the line connecting the current and next trace points. $\mathbf{W}_{k}$ and $\mathbf{V}_{k}$ are the system noise and the observation noise, respectively.

In grouping of blood vessels, the relationships between the seven state variables are defined as follows:

$$
\begin{aligned}
\mathbf{X}_{k}= & f_{k-1}\left(\mathbf{X}_{k-1}\right)+\mathbf{W}_{k-1}= \\
= & {\left[\begin{array}{c}
x_{k-1}+d_{k-1} \times \cos \theta_{k-1} \\
y_{k-1}+d_{k-1} \times \sin \theta_{k-1} \\
G_{k-1}+\dot{G}_{k-1} \\
d_{k-1}+\dot{d}_{k-1} \\
\theta_{k-1} \\
\dot{G}_{k-1} \\
\dot{d}_{k-1}
\end{array}\right]+\left[\begin{array}{c}
0 \\
0 \\
0 \\
0 \\
w_{1} \\
w_{2} \\
w_{3}
\end{array}\right] . }
\end{aligned}
$$

$w_{1}, w_{2}$ are the system errors, which mainly come from the unpredictable vessel direction change and the quantization error of intensity values. $w_{3}$ is the system error as the actual positions of image points are discretized into integral numbers of pixels. $w_{i}$ are assumed to be mutually independent, zero mean white Gaussian noise with a covariance matrix $\mathbf{Q}$, which is determined as follows:

$$
\mathbf{Q}=\left[\begin{array}{ccccccc}
0 & 0 & 0 & 0 & 0 & 0 & 0 \\
0 & 0 & 0 & 0 & 0 & 0 & 0 \\
0 & 0 & 0 & 0 & 0 & 0 & 0 \\
0 & 0 & 0 & 0 & 0 & 0 & 0 \\
0 & 0 & 0 & 0 & 0.2 & 0 & 0 \\
0 & 0 & 0 & 0 & 0 & 1.0 & 0 \\
0 & 0 & 0 & 0 & 0 & 0 & 1.0
\end{array}\right]
$$


The nonzero entries are estimated using the 20 training images in the DRIVE dataset. The higher the covariance values, the stronger the influence of the observation measurements will be.

The state variables are related to the observation variables by

$$
\mathbf{X}_{k}^{o}=h_{k}\left(\mathbf{X}_{k}\right)+\mathbf{V}_{k}=\left[\begin{array}{c}
x_{k} \\
y_{k} \\
G_{k} \\
d_{k} \\
\theta_{k}
\end{array}\right]+\left[\begin{array}{c}
v_{1} \\
v_{2} \\
v_{3} \\
v_{4} \\
v_{5}
\end{array}\right]
$$

where $v_{1}, \ldots, v_{4}$, and $v_{5}$ are observation errors arising primarily from the vessel tracer. $v_{i}$ are assumed to be mutually independent and zero mean white Gaussian noise with a covariance matrix $\mathbf{R}$, which is determined as follows:

$$
\mathbf{R}=\left[\begin{array}{ccccc}
1 & 0 & 0 & 0 & 0 \\
0 & 1 & 0 & 0 & 0 \\
0 & 0 & 1 & 0 & 0 \\
0 & 0 & 0 & 1 & 0 \\
0 & 0 & 0 & 0 & 0.2
\end{array}\right] .
$$

$\mathbf{R}(i, i)$ for $i=1 . .4$ are set to 1 since the measurements of centerline locations, color intensity, and vessel width are quantified to the nearest integers. $\mathbf{R}(5,5)$ for $\theta_{k}$ is obtained from $\mathbf{Q}(5,5)$. Opposite to $\mathbf{Q}$, the smaller the covariance values, the stronger the influence of the observation measurements will be.

The Kalman theory gives the equations for the optimal estimate $\hat{\mathbf{X}}_{k \mid k-1}$ of $\mathbf{X}_{k}$ given the statistics of the system and observation noise from $\hat{\mathbf{X}}_{k-1 \mid k-1}$. The Kalman filter has two distinct phases: predict and update. In the predict phase, the previous state estimate $\hat{\mathbf{X}}_{k-1 \mid k-1}$ is used to produce an estimate of the current state $\hat{\mathbf{X}}_{k \mid k-1}$. The a priori state estimate and the estimate covariance are computed, respectively, as

$$
\begin{aligned}
\hat{\mathbf{X}}_{k \mid k-1} & =f_{k-1}\left(\hat{\mathbf{X}}_{k-1 \mid k-1}\right) \\
\mathbf{P}_{k \mid k-1} & =A_{k} \mathbf{P}_{k-1 \mid k-1} A_{k}^{T}+\mathbf{Q} .
\end{aligned}
$$

where the matrix $A_{k}$ is defined as $\partial f_{k-1}\left(\mathbf{X}_{k-1}\right) / \partial \mathbf{X}_{k-1}$ :

$$
\begin{aligned}
A_{k} & =\frac{\partial f_{k-1}\left(\mathbf{X}_{k-1}\right)}{\partial \mathbf{X}_{k-1}} \\
& =\left[\begin{array}{llllcll}
1 & 0 & 0 & \cos \theta_{k-1} & -d \times \sin \theta_{k-1} & 0 & 0 \\
0 & 1 & 0 & \sin \theta_{k-1} & d \times \cos \theta_{k-1} & 0 & 0 \\
0 & 0 & 1 & 0 & 0 & 1 & 0 \\
0 & 0 & 0 & 1 & 0 & 0 & 1 \\
0 & 0 & 0 & 0 & 1 & 0 & 0 \\
0 & 0 & 0 & 0 & 0 & 1 & 0 \\
0 & 0 & 0 & 0 & 0 & 0 & 1
\end{array}\right] .
\end{aligned}
$$

In the update phase, the current a priori prediction is combined with the current observation information to refine the state estimate. The a posteriori state estimate and the estimate covariance are computed, respectively, as

$$
\begin{aligned}
\hat{\mathbf{X}}_{k \mid k} & =\hat{\mathbf{X}}_{k \mid k-1}+\mathbf{K}_{k}\left(\mathbf{X}_{k}^{o}-h_{k}\left(\hat{\mathbf{X}}_{k \mid k-1}\right)\right) \\
\mathbf{P}_{k \mid k} & =\left(I-\mathbf{K}_{k} \mathbf{H}_{k}\right) \mathbf{P}_{k \mid k-1}
\end{aligned}
$$

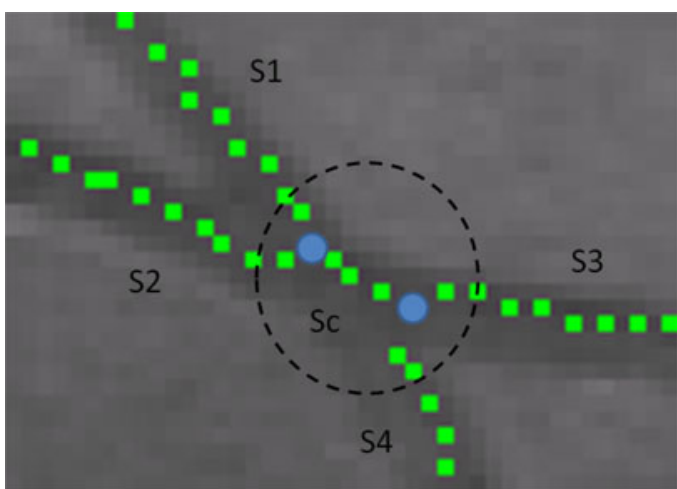

Fig. 7. Incorrect tracing, resulting in splitting of one crossing into two branches. The system resolves this type of conflict by performing minimum-cost matching. With minimum-cost matching, $S_{1}, S_{2}, S_{c}$, and $S_{4}$ are regrouped to maximize the total continuity at the junction.

where the optimal Kalman gain $\mathbf{K}_{k}$ is defined as

$$
\mathbf{K}_{k}=\mathbf{P}_{k \mid k-1} \mathbf{H}_{k}^{T}\left(\mathbf{H}_{k} \mathbf{P}_{k \mid k-1} \mathbf{H}_{k}^{T}+\mathbf{R}\right)^{-1} .
$$

The matrix $\mathbf{H}_{k}$ is defined as $\partial h_{k}\left(\mathbf{X}_{k}\right) / \partial \mathbf{X}_{k-1}$ :

$$
\mathbf{H}_{k}=\frac{\partial h_{k}\left(\mathbf{X}_{k}\right)}{\partial \mathbf{X}_{k-1}}=\left[\begin{array}{ccccccc}
1 & 0 & 0 & 0 & 0 & 0 & 0 \\
0 & 1 & 0 & 0 & 0 & 0 & 0 \\
0 & 0 & 1 & 0 & 0 & 0 & 0 \\
0 & 0 & 0 & 1 & 0 & 0 & 0 \\
0 & 0 & 0 & 0 & 1 & 0 & 0
\end{array}\right] \text {. }
$$

For each vessel, $\hat{\mathbf{X}}_{0}$ is obtained from the first trace point of the root segment given by the LRV tracer with $\dot{G}_{0}, \dot{d}_{0}$ set to 1. $\theta_{0}$ is the vessel direction obtained from the first and second trace points. $P_{0}$ is the diagonal matrix containing the estimated deviation of the seven state variables:

$$
P_{0}=\left[\begin{array}{ccccccc}
1 & 0 & 0 & 0 & 0 & 0 & 0 \\
0 & 1 & 0 & 0 & 0 & 0 & 0 \\
0 & 0 & 1 & 0 & 0 & 0 & 0 \\
0 & 0 & 0 & 1 & 0 & 0 & 0 \\
0 & 0 & 0 & 0 & 0.5 & 0 & 0 \\
0 & 0 & 0 & 0 & 0 & 1 & 0 \\
0 & 0 & 0 & 0 & 0 & 0 & 1
\end{array}\right] .
$$

When the Kalman filter reaches the end of a vessel segment, $\mathbf{X}_{k}^{o}$ is chosen from the segments that come to meet at this junction in the search range of $\hat{\mathbf{X}}_{k \mid k-1}{ }^{1}$, and the segment in which $\mathbf{X}_{k}^{o}$ resides is the one to be connected to the current segment. $\mathbf{X}_{k}^{o}$ is the state vector of the first trace point of the neighboring segment with the minimum Mahalanobis distance defined as follows:

$$
\begin{aligned}
& d\left(h_{k}\left(\hat{\mathbf{X}}_{k \mid k-1}\right), \mathbf{X}_{k}^{o}\right) \\
& \quad=\sqrt{\left(h_{k}\left(\hat{\mathbf{X}}_{k \mid k-1}\right)-\mathbf{X}_{k}^{o}\right)^{T} \Sigma^{-1}\left(h_{k}\left(\hat{\mathbf{X}}_{k \mid k-1}\right)-\mathbf{X}_{k}^{o}\right)} .
\end{aligned}
$$

\footnotetext{
${ }^{1}$ The search range is defined to be $m$ pixels away from $\hat{\mathbf{X}}_{k \mid k-1}$, where $m$ is the maximum vessel width of the image.
} 
TABLE I

COMPONENT ANALYSIS: SuCCESS RATES FOR 20 IMAGES FrOM THE DRIVE DATASET

\begin{tabular}{|r||r|r|r|r|r|r|r|r|}
\hline \multicolumn{1}{|c||}{} & \multicolumn{4}{c|}{ LRV Tracing } & \multicolumn{4}{c|}{ Modified LRV Tracing } \\
& \multicolumn{1}{|c|}{ (1829 segments/63718 trace points) } & \multicolumn{2}{c|}{ (1874 segments/68852 trace points) } \\
\hline \hline Experiment & 1 & 2 & 3 & 4 & 1 & 2 & 3 & 4 \\
\hline Success rates(\%) & 75.34 & 79.06 & 76.00 & 81.14 & 78.23 & 85.06 & 81.70 & 88.79 \\
\hline Standard deviation(\%) & 3.08 & 3.88 & 3.26 & 3.92 & 3.77 & 5.60 & 4.33 & 4.30 \\
\hline
\end{tabular}

The covariance matrix $\Sigma$ was obtained using the 20 training images. Finally, the corrected state vector $\hat{\mathbf{X}}_{k \mid k}$ becomes the first state vector of the new segment. The grouping process for the current vessel terminates if no ungrouped segment is found in the search range of $\hat{\mathbf{X}}_{k \mid k-1}$.

\section{B. Improving Continuity Using Minimum Cost Matching}

During the grouping process, the chosen segment with the minimum Mahalanobis distance may already be connected to another vessel. The system resolves this type of conflict by performing minimum-cost matching [30]. This problem can be formulated as a weighted bipartite matching problem. In a graph representation, the vessel segments are the colored vertices and the Mahalanobis distance $d($.$) between two segments is the$ weight assigned to the edge connecting two vertices. To assign the colors to the vertices (vessel segments) in the graph, the current vessel segment is assigned color $c_{1}$, and all its potential successors are assigned color $c_{2}$. If the successor $C$ with the least $d($.$) value is already grouped, label the current parent$ segment of $C$ the color $c_{1}$, and all the potential successors of the parent segment are colored $c_{2}$ as well. Edges are defined between every vertex of $c_{1}$ and vertex of $c_{2}$. The minimum-cost matching problem can be easily solved using the maximal flow algorithm [31] by connecting all vertices of $c_{1}$ to a super source vertex and connecting vertices of $c_{2}$ to a super sink vertex.

Fig. 7 shows an example of minimum-cost matching. $S_{1}, S_{c}$ are first connected before grouping of $S_{2}$ takes place. When choosing the next segment for grouping at the end point of $S_{2}$, $S_{c}$ is again chosen as the next candidate segment because it has the highest continuity with $S_{2}$ among segments in the search range of $S_{2}$. Both $S_{1}$ and $S_{2}$ are given color $c_{1}$, and $S_{c}$ and $S_{4}$ given color $c_{2}$ ( $S_{3}$ is not considered in this example since it is already connected to $S_{c}$.) With minimum-cost matching, instead of choosing the second candidate $S_{4}$ for $S_{2}, S_{1}, S_{2}, S_{c}$, and $S_{4}$ are regrouped to maximize the total continuity-which is to minimize the sum of the Mahalanobis distances-at the junction. If grouping of $S_{1}$ with $S_{4}$ and $S_{2}$ with $S_{c}$ has higher total continuity than grouping of $S_{1}$ with $S_{c}$ and $S_{2}$ with $S_{4}$, the former will be accepted as the new grouping result. Otherwise, grouping of $S_{1}$ and $S_{c}$ will remain intact and $S_{4}$ will be grouped to $S_{2}$.

\section{EXPERIMENTAL ANALYSIS}

The proposed system trained and tested using the DRIVE dataset [32]. The first 20 images were used to train the Kalman filter system model and to estimate the Mahalanobis distance covariance matrix. The remaining 20 images were used to test the system, and the set consists of 17 relatively normal images and 3

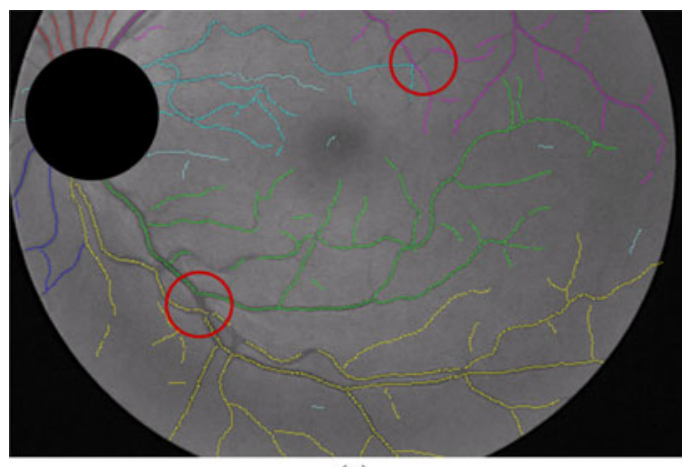

(a)

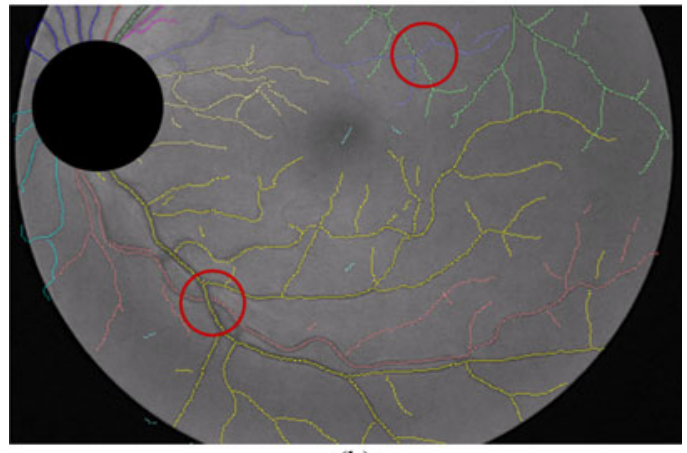

(b)

Fig. 8. Improvement on vessel extraction over the original LRV algorithm for tree reconstruction. (a) Result of LRV tracing with the threshold value of 2.0 as set in [5]. (b) Result of our improved tracing algorithm. The red circles indicate vessels discarded by the original LRV algorithm.

pathological images of various degree of severity. Images were acquired using a Canon CR6 nonmydriatic 3CCD camera at a 45 field-of-view and are 24-bit color at a resolution of $768 \times 584$ (the fundus occupies a circle of diameter approximately 540 pixels about $50 \%$ of the image).

The results of vascular tree reconstruction were compared against the groundtruth images provided by a physician. Figs. 9(a) and 11(b) are examples of groundtruth images. Each retinal vascular tree is assigned a nonwhite color such that trees that cross are of different colors. An area is painted white if more than one vessel passes through it. A segment is painted gray if it does not belong to any retinal vascular tree. Such vessels are choroidal vessels in the choroidal layer, as seen in Fig. 11(b), and are not considered, since the proposed system aims to reconstruct retinal vascular trees only. ${ }^{2}$

In our performance analysis, a segment is correctly connected to its parent segment if its color in the groundtruth image is the same as its parent segment. This study defines the success

\footnotetext{
${ }^{2}$ Because of thinning of the pigmented epithelial layer above it, choroida vessels can appear as fragmented vessels in a retinal image.
} 


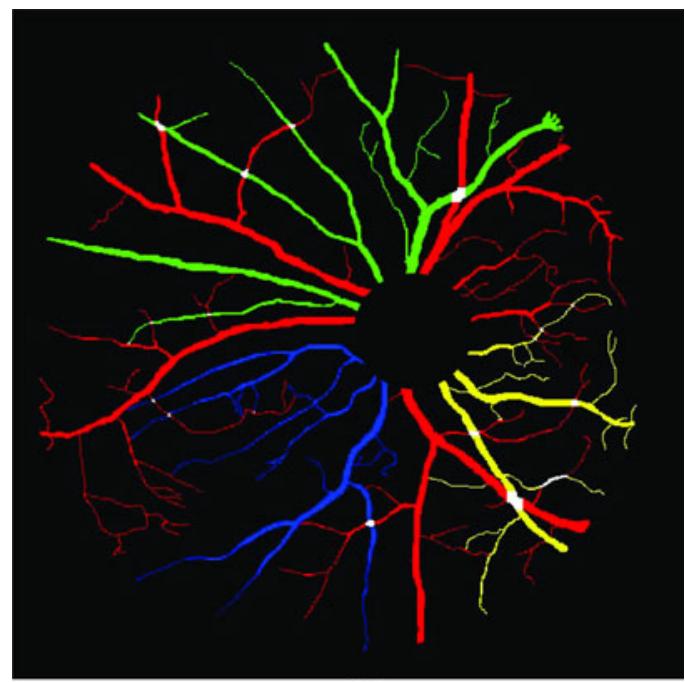

(a)

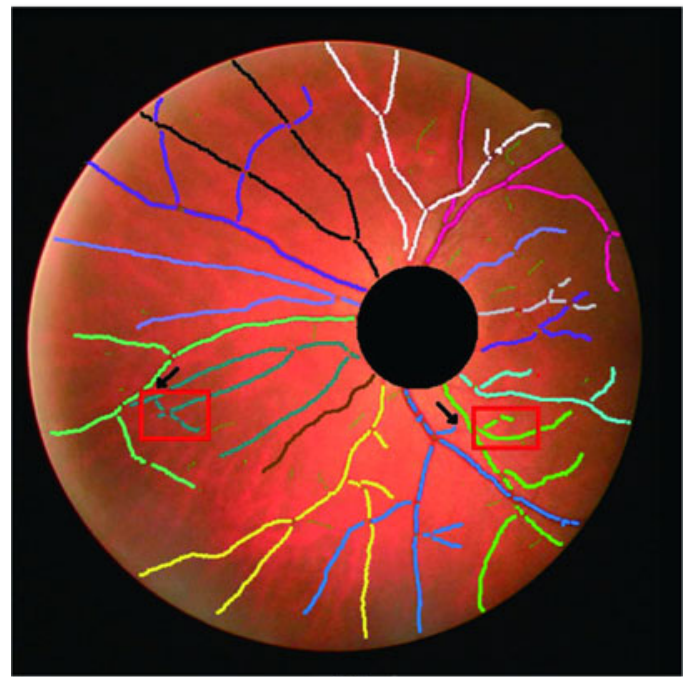

(b)

Fig. 9. Best case in our test suite with a success rate of 95\%. (a) Vessel tree groundtruth. (b) Result of vessel tree reconstruction. The red boxes highlight the incorrectly classified segments due to missing traces.

rate as the percentage of correctly connected segments over the total number of segments involved in the reconstruction process. The total number of segments does not include the false positive segments, such as the choroidal vessels. However, if a true positive segment is connected to a false positive segment, it is treated as an incorrectly connected segment.

\section{A. Component Analysis on Vessel Grouping}

To isolate the effectiveness of each major component in the proposed tree reconstruction algorithm, this study includes the performance analysis on four variations. These variations used the results of LRV tracing and the results of the modified LRV, respectively. There were 1829 segments (63718 trace points) extracted using LRV tracing with the threshold value 2.0 as set in [5] and 1874 segments (68 852 trace points) extracted using the modified LRV. Given the trace results, the four variations on tree reconstruction are as follows.

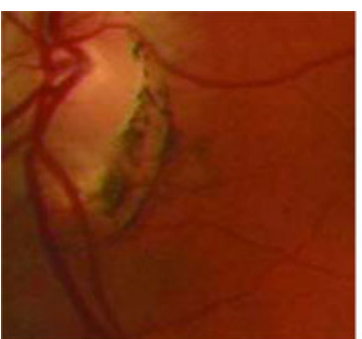

(a)

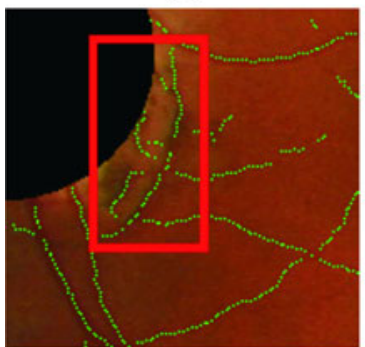

(c)

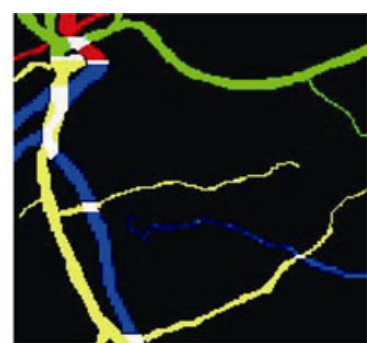

(b)

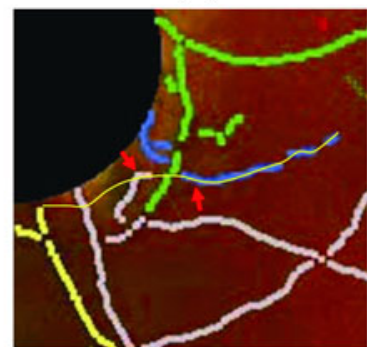

(d)
Fig. 10. Case of incorrect grouping due to erroneous tracing of the pathological structures. (a) Original image. (b) Vessel tree groundtruth. (c) Red box highlighting the area with false positive traces. (d) Blue line is the result of incorrect grouping, whereas the thin yellow line is drawn manually to indicate the correct grouping.

1) Grouping without the involvement of the Kalman filter and minimum-cost matching.

2) Grouping with the Kalman filter, but without minimumcost matching by considering only the ungrouped segments when choosing the next segment at the junction.

3) Grouping without the Kalman filter but with minimumcost matching if a conflict arises at the junction.

4) Our proposed grouping method (with both the Kalman filter and minimum-cost matching).

Table I summarizes the grouping success rates of the four variations. One can observe that the first variation yields the lowest success rate, regardless of tracing methods. This is primarily due to the fact that certain vessel properties (e.g., width, direction, etc.) are less reliable near junction points. For the second variation, the Kalman filter is applied to improve the continuity of vessel segments at bifurcation or crossover points. Consequently, the success rate is improved as compared to that of the first variation. For the third variation, minimum cost matching is utilized as an attempt to repair incorrect tracing at junction points. The resulting success rate is better than that of the first variation but worse than that of the second variation. Regardless of tracing methods, the highest success rate is achieved by synergetic use of the Kalman filter and minimum cost matching, i.e., the fourth variation. The basic idea behind this scheme is to perform preliminary grouping (i.e., Kalman filtering) and then repair incorrectly grouped vessel segments (i.e., minimum cost matching). In Table I, the results clearly demonstrate that the addition of minimum-cost matching further improves our previous method [6], [7], i.e., the second variation.

The efficacy of grouping also depends on the robustness of the tracing algorithm-our modified LRV method enhanced the grouping results by $9.43 \%(88.79 / 81.14)$ compared to the 


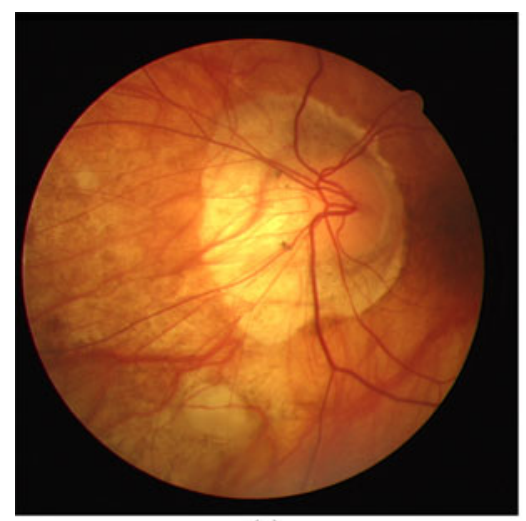

(a)

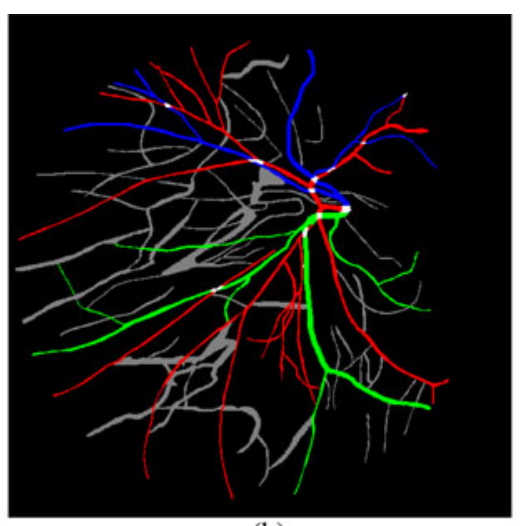

(b)

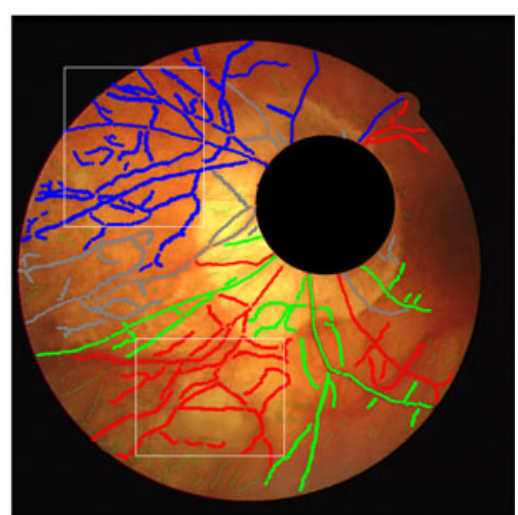

(c)

Fig. 11. Worst case in our suite, with a success rate of $75.38 \%$. Many retinal vessels were connected to the choroidal vessels by mistake. (a) Original image. (b) Vessel tree groundtruth. (c) Result of vessel tree reconstruction.

original LRV algorithm. To qualitatively demonstrate the improvement over the LRV algorithm on vessel extraction, Fig. 8(a) shows the grouping result from the original LRV tracing, whereas Fig. 8(b) shows the grouping results from the improved algorithm. The red circles in Fig. 8(a) indicate vessels that were discarded by the original LRV algorithm even with the threshold value set to 1 . The reason that segments shown in the circles are incorrectly grouped is inaccurate tracing, which turns crossovers to bifurcations. Overall, the improved LRV tracer increases the number of trace points considerably, with the majority of them being true positive. The improvement here is $8.1 \%$ (68 852/63718) in the number of true positive trace points. The same effect cannot be achieved by simply decreasing the threshold on the LRV value, which increases false positive trace points at a much higher rate than the true positive trace points.

Fig. 9 shows the best case, with a success rate of $95 \%$. The retinal image is relatively healthy with a well-defined vasculature. However, the efficacy of grouping depends heavily on the robustness of the tracing algorithm. The segments shown in the boxes in Fig. 9(b) are incorrectly grouped due to missing traces, which turn crossovers to bifurcations (as pointed out by the arrows). Fig. 10 shows a difficult case (low severity pathological) in which some segments are obtained from pathological structures. The thin yellow line indicated by the arrows in Fig. 10(d) is drawn manually to indicate the correct grouping if tracing were performed correctly for the thin vessel. Tracing was corrupted due to the presence of lesions near the optic disk. Fig. 11shows the case with the lowest success rate of $75.38 \%$. This is a case of severe myopia with visible choroidal blood vessels. Since the vessel tracer detected both retinal and choroidal vessels, the success rate for retinal vessel construction can be substantially reduced if retinal vessels are accidentally connected to the choroidal vessels.

Both LRV and tensor voting enhancement are much more computationally expensive than grouping, since the former are implemented as convolution with a set of templates. In vessel grouping, the execution time is based on the number of vessel segments found. Running on a computer with AMD Athlon 64 Processor and 2G RAM, the average execution time for a $584 \times 584$ image is approximately $4 \mathrm{~min}$ for LRV vessel tracing

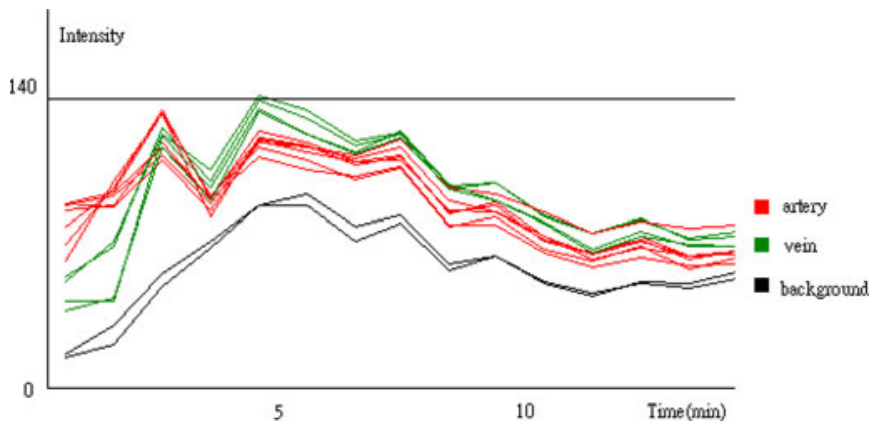

Fig. 12. Temporal intensity profiles of sampled points from the arterial trees, venous trees and the background. If the intensity profile is available, it replaces the green channel to provide better distinction between arterial and venous vessels at crossovers.

and 2 min for tensor voting. For vessel grouping, the execution time goes as low as $35 \mathrm{~s}$ for 67 vessel segments and as high as $92 \mathrm{~s}$ for 126 segments.

\section{B. Generalization of the Algorithm}

To generalize vessel grouping for different datasets taken under different imaging conditions or with special physiological characteristics, sample images should be used to train the system to obtain data-dependent parameters, which are $\mathbf{Q}$ (the covariance matrix for the system errors) and $\mathbf{R}$ (the covariance matrix for the observation errors) in our system. To obtain the variances for $\theta_{k}, \dot{G}_{k}$, and $\dot{d}_{k}$ (the nonzero diagonal elements in $\mathbf{Q}$ ), the traced vessel segments in the training images are used to obtain the standard deviations of vessel tangent direction $\theta_{k}$, change in vessel intensity $\dot{G}_{k}$, and change in vessel width $\dot{d}_{k}$. For the DRIVE dataset, the numbers are $0.4421,1.0122$, and 1.0034, resulting in variances of $0.2,1$ and 1 , respectively. For $\mathbf{R}$, the only data-dependent element is $\mathbf{R}(5,5)$ (the variance on $\theta_{k}$ ), and it is set to the same value as $\mathbf{Q}(5,5)$.

The proposed grouping method can be easily modified to take advantage of the temporal information provided by a fluorescein angiogram (FA) sequence to improve tree reconstruction. FA is a special kind of photograph often used by physicians for treatment of vascular-related diseases, since vessels obscured by 


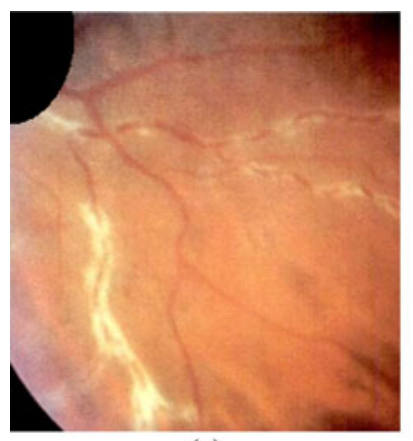

(a)

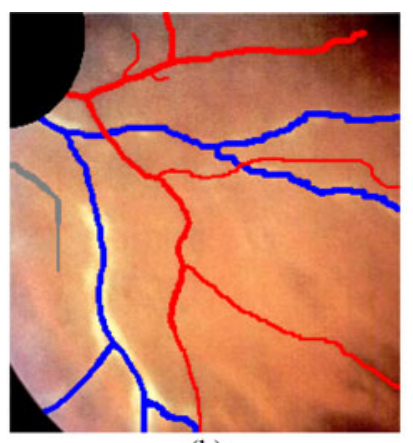

(b)

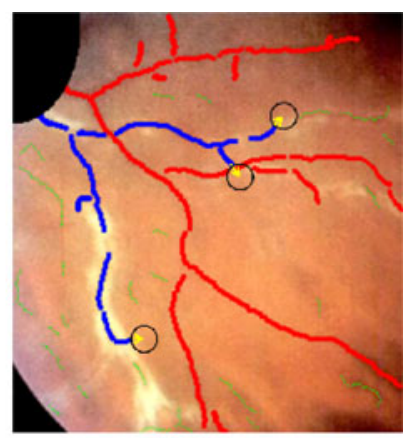

(c)

Fig. 13. Sheathing of retinal artery of retinal vasculitis with nonperfusion vessels. (a) Original image. (b) Vessel tree groundtruth. (c) Result of vessel tree reconstruction. The tip of a yellow arrow indicates the Kalman filter estimation of the next vessel point. No potential successor segment is found in the search range, indicated as the black circle, due to either disappearance of sections of a vessel or substantial changes in width and curvature (high tortuosity).

lesions can be highlighted by the fluorescein dye. Instead of using a single green channel from the color image, the feature $G$ is replaced with the temporal intensity profile $I=\left\{I_{1}, I_{2}, \ldots, I_{n}\right\}$ of a trace point, where $n$ is the number of aligned FA images ${ }^{3}$ in the sequence. Since fluorescein dye enters the ocular circulation from arteries to veins, this accounts for the delay between arterial stage phase and veneous phase [34]. As a result, the temporal intensity profile of an arterial trace point will increase and fade away earlier than that of a venous trace point. This phenomenon can be clearly observed with the representative profiles shown in Fig. 12. This property is important for grouping at crossovers because arterial trees only cross with venous trees.

This study includes an experiment using six FA sequences. The dataset was obtained from the Taipei Veterans General Hospital and all sequences were pathological. Each FA sequence consists of a color retinal image and over six FA images. The image size is $800 \times 800$. The results of the vascular tree reconstruction were compared with the groundtruth images provided by a physician. A total of 444 segments (22547 trace points) was extracted from a suite of six sequences. If the intensity feature is represented using only the green channel of the color image, the average success rate is $83.11 \%^{4}$, with a standard deviation of $3.63 \%$. If replacing the green channel with the intensity profile from the complete FA sequence, the average success rate is boosted up to $90.09 \%$, and the standard deviation decreases to $1.15 \%$, achieving much more consistent performance. With the fluorescein information, the likelihood of grouping an artery with a vein, or vice versa, is substaintially reduced.

\section{Comparison With an Existing Algorithm}

We compared our work to [21] by Al-Diri et al., which has the most similar goal as our work. Using the definition in [21], the success is measured using "joining forms," which include all the junctions, bridges, and leave segments. For the DRIVE dataset, Al-Diri's method extracts 4023 joining forms, of which 1011 are junctions/bifurcations, 2112 are bridges (including crossovers),

\footnotetext{
${ }^{3}$ This study aligns the images of an FA sequence into a common space The registration technique used in this study is the edge-driven dual bootstrap iterative closest point algorithm [33].

${ }^{4}$ The average success rate for the FA dataset is lower than for the DRIVE dataset since the FA datast contains only pathological sequences.
}

and 900 are leaves. The overall success rate and the success rates for the three types of joint form are $71 \%, 71 \%, 76 \%$, and $59 \%$, respectively. Our method extracts 2578 joining forms in total with 821 junctions, 524 bridges, and 1233 leaves. The overall success rate and the success rates for the three types of joint form are $83 \%, 76 \%, 83 \%$, and $88 \%$, respectively. It is interesting to notice that Al-Diri's method produces far more junctions and bridges, but much fewer leaves than our method does. One of the reasons is that many segments which should be labeled as leaves are incorrectly connected to other trees and each becomes part of a junction or a bridge using Al-Diri's method. Another reason for the high number in bridges by Al-Diri's method is that more fragmented segments are generated and connected as bridges. Overall, our method outperforms Al-Diri's method by $17 \%$.

\section{DISCUSSION AND CONCLUSION}

This study describes an approach that improves on an existing vessel segmentation algorithm and performs grouping on the extracted vessel segments to restore the topology of vascular trees with anatomical realism. If an operator can provide the tree type (artery/vein), trees extracted using our proposed system can facilitate the quantification of geometrical and topological properties of veins and arteries for the study of certainly medical conditions, such as arteriolar narrowing and hypertension.

For vessel segmentation, both vesselness and connectedness are exploited to maximize the completeness of vessel extraction. For vessel grouping, the Kalman filter is adopted to ensure continuity of vessel segments at the bifurcation and crossover points. Minimum-cost matching is utilized to correct inaccurate tracing of junction points. Note that the existing approaches [3], [16]-[23] do not model the changes of vessel properties within a vessel segment. However, this information can be used to improve the reliability of performing tracing at vessel junctions. Using the extended Kalman filter, it is possible to model the continuous changes in curvature, width, and intensity of vessel segments, and to more accurately connect those segments at crossovers and bifurcations.

The average success rates in this study are $88.79 \%$ for color retinal images from the DRIVE dataset and $90.09 \%$ for pathological FA sequences. The efficacy of grouping depends heavily on the robustness of the tracing algorithm since incorrect 
grouping is likely to occur at places where vessels are missed or pathological structures are mistaken as vessels. Challenges for grouping alone come from medical conditions with nonperfusion vessels, such as sheathing of retinal artery of retinal vasculitis (see Fig. 13(a) [35] for an example). Due to nonperfusion resulting from blockage or inflammation, vessels can appear disconnected and have undergone substantial changes in width and tortuosity. Even with correct tracing, our system is not yet capable of handling such changes since it assumes connected vessels with continuity in geometric properties. Disconnected vessels can be out of the search range of one another, and substantial changes in vessel properties can promote the system to connect the current segment to another segment from a different vessel with higher continuaty [see Fig. 13(c)]. In the next phase of development, we will focus on elimination of false vessels, which is the major reason for failure in grouping, and proper handling of nonperfusion vessls to improve the clinical usability of the system.

\section{REFERENCES}

[1] M. Sonka, A. Stolpen, W. Liang, and R. M. Stefancik, "Vascular imaging and analysis," in Handbook of Medical Imaging, Medical Image Processing and Analysis, vol. 2, Bellingham, WA: SPIE Press, ch. 14, 2001, p. 809 V914

[2] C. S. Ogilvy, B. S. Carter, S. Kaplan, C. Rich, and R. M. Crowell, "Temporary vessel occlusion for aneurysm surgery: Risk factors for stroke in patients protected by induced hypothermia and hypertension and intravenous mannitol administration," J. Neurosurg., vol. 84, no. 5, pp. 785-791, May 1996.

[3] D. Selle, B. Preim, A. Schenk, and H.-O. Peitgen, "Analysis of vasculature for liver surgical planning," IEEE Trans. Med. Imaging., vol. 21, no. 11, pp. 1344-1357, Nov. 2002.

[4] A. Stanton, B. Wasan, A. Cerutti, S. Ford, R. Marsh, P. Sever, S. Thom, and A. Hughes, "Vascular network changes in the retina with age and hypertension," J. Hypertension, vol. 13, no. 12, pp. 1724-1728, 1995.

[5] M. Sofka and C. V. Stewart, "Retinal vessel centerline extraction using multiscale matched filters, confidence and edge measures," IEEE Trans. Med. Imaging., vol. 25, no. 12, pp. 1531-1546, Dec. 2006.

[6] K.-S. Lin, C.-L. Tsai, M. Sofka, C.-H. Tsai, S.-J. Chen, and W.-Y. Lin, "Vascular tree construction with anatomical realism for retinal images," In 9th IEEE Int. Conf. BioInf. BioEng, Jun. 2009, pp. 313-318

[7] K.-S. Lin, P.-Y. Yeh, C.-L. Tsai, S.-J. Chen, and W.-Y. Lin, "Retinal vascular tree construction with multimodal fluorescein angiogram sequence," Biomed. Eng.: Appl. Basis Commun., vol. 22, pp. 101-110, 2010.

[8] A. D. Hughes, E. Martinez-Perez, A.-S. Jabbar, A. Hassan, N. W. Witt, P. D. Mistry, N. Chapman, A. D. Stanton, G. Beevers, R. Pedrinelli, K. Parker, and S. Thom, "Quantification of topological changes in retinal vascular architecture in essential and malignant hypertension," J. Hypertension, vol. 24, pp. 889-894, 2006.

[9] N. Patton, T. M. Aslam, T. MacGillivray, I. J. Deary, B. Dhillon, R. H. Eikelboom, K. Yogesan, and I. J. Constable, "Retinal image analysis: Concepts, applications and potential," Prog. Retinal Eye Res., vol. 25, pp. 99-127, 2006.

[10] H. M. Pakter, E. Ferlin, S. C. Fuchs, M. K. Maestri, R. S. Moraes, G. Nunes, L. B. Moreira, M. Gus, and F. D. Fuchs, "Measuring arteriolarto-venous ratio in retinal photography of patients with hypertension: Development and application of a new semi-automated method," Amer. J. Hypertension, vol. 18, no. 3, pp. 417-421, 2004.

[11] H. F. Jelinek and M. J. Cree, Automated Image Detection of Retinal Pathology. Boca Raton, FL: CRC Press, 2009.

[12] T. Y. Wong, L. D. Hubbard, E. K. Marino, R. Kronmal, A. R. Sharrett, D. S. Siscovick, G. Burke, and J. M. Tielsch, "Retinal microvascular abnormalities and blood pressure in older people: The cardiovascular health study," Brit. J. Ophthal., vol. 86, no. 9, pp. 1007-1013, 2002.

[13] S. Chatterjee, S. Chattopadhya, M. Hope-Ross, and P. Lip, "Hypertension and the eye: Changing perspectives," J. Hum. Hypertens., vol. 16, pp. 667$675,2002$.
[14] M. E. Martinez-Perez, A. D. Hughes, A. V. Stanton, S. A. Thom N. Chapman, A. A. Bharath, and K. H. Parker, "Retinal vascular tree morphology: A semi-automatic quantification," IEEE Trans, Biomed, Eng,, vol. 49, no. 8, pp. 912-917, Aug. 2002.

[15] L. Hubbard, R. Brothers, W. King, L. Clegg, R. klein, L. Cooper, R. Sharrett, M. Davis, and J. Cai, "Methods for evaluation of retinal microvascular abnormalities associated with hypertension/sclerosis in the atherosclerosis risk in communities study," Ophthalmology, vol. 106, no. 12, pp. 2269-2280, 1999.

[16] P. J. Yim, P. L. Choyke, and R. M. Summers, "Gray-scale skeletonization of small vessels in magnetic resonance angiography," IEEE Trans. Med. Imaging., vol. 19, no. 6, pp. 568-576, Jun. 2000.

[17] K. Haris, S. N. Efstratiadis, N. Maglaveras, C. Pappas, J. Gourassas, and G. Louridas, "Model-based morphological segmentation and labeling of coronary angiograms," IEEE Trans. Med. Imaging., vol. 18, no. 10, pp. 1003-1015, Oct. 1999.

[18] I. Liu and Y. Sun, "Recursive tracking of vascular networks in angiograms based on the detection-deletion scheme," IEEE Trans. Med. Imaging., vol. 12, no. 2, pp. 334-341, Jun. 1993.

[19] J. Jomier, V. LeDigarcher, and S. Aylward, "Automatic vascular tree formation using the mahalanobis distance," In Proc. 8th Med Image Comput. Comput. Assist. Interv., Palm-Springs, CA, 2005, pp. 806-812

[20] E Bullitt, S Aylward, A. Liu, J. Stone, S. Mukherji, C. Coffey, G. Gerig, and S. M. Prizer, "3d graph description of the intracerebral vasculature from segmented MRA and tests of accuracy by comparison with X-ray angiograms," in Information Processing in Medical Imaging (Lecture Notes in Computer Science Series), vol. 1613, Visegrád, Hungary: SpringerVerlag, Jun./Jul. 1999, pp. 308-320

[21] B. Al-Diri, A. Hunter, D. Steel, and M. Habib, "Automated analysis of retinal vascular network connectivity," Comp. Med. Imag. Graph., pp. 462-470, 2010

[22] P. Montesinos, L. Alquier, Sci. G. Parc, and N. Besse, "Perceptual organization of thin networks with active contour functions applied to medical and aerial images," In Int. Conf. Pattern Recogn., 1996, vol. 1, pp. 647-651

[23] G. Coppini, M. Demi, R. Poli, and G. Valli, "An artificial vision system for x-ray images of human coronary tree," IEEE T. Pattern Anal., vol. 15, no. 2, pp. 156-162, Feb. 1993.

[24] T. Deschamps and L. D. Cohen, "Grouping connected components using minimal path techniques. Mathematics visualization," in Geometric Methods in Bio-Medical Image Processin, Berlin, Germany: Springer, 2002.

[25] T. Deschamps and L. D. Cohen, "Grouping connected components using minimal path techniques. Application to reconstrucion of vessels in $2 \mathrm{C}$ and 3d images," in Proc. Comput. Vision Pattern Recogn., 2001, vol. 2, pp. $102-109$.

[26] S. M. Kay, Fundamentals of Statistical Signal Processing: Detection Theory. Englewood Cliffs, NJ: Prentice Hall, 1998.

[27] G. Guy and G. Medioni, "Interring global perceptual contours from local features," Int. J. Comput. Vis., vol. 20, no. 1, pp. 113-133, 1996.

[28] A. Neubeck and L. J. Van Gool, "Efficient non-maximum suppression," in Proc. Int. Conf. Pattern Recog, 2006, pp. 850-855.

[29] A. Hoover, V. Kouznetsova, and M. Goldbaum, "Locating blood vessels in retinal images by piecewise threshold probing of a matched filter response," IEEE Trans. Med. Imaging., vol. 19, no. 3, pp. 203-210, Mar. 2000.

[30] S. R. Buss and P. N. Yianilos, "Linear and o(n $\log n)$ time minimum-cost matching algorithms for quasi-convex tours," in Proc. 5th Annu. ACMSIAM Symp. Discrete Algorithms, 1994, pp. 65-76.

[31] N. Deo, Graph Theory With Applications to Engineering and Computer Science. Englewood Cliffs, NJ: Prentice-Hall, 1974.

[32] J. J. Staal, M. D. Abramoff, M. Niemeijer, M. A. Viergever, and B. van Ginneken, "Ridge based vessel segmentation in color images of the retina," IEEE Trans. Med. Imaging., vol. 23, no. 4, pp. 501-509, Apr. 2004.

[33] C.-L. Tsai, C.-Y. Li, G. Yang, and K.-S. Lin, "The edge-driven dualbootstrap iterative closest point algorithm for registration of multimodal fluorescein angiogram sequence," IEEE Trans. Med. Imaging., vol. 29, no. 3, pp. 636-649, Mar. 2010.

[34] A. Agarwal, Fundus Fluorescein and Indocyanine Green Angiography: A Textbook and Atlas. Thorofare, NJ: Slack, 2007.

[35] H. Tabandeh and M. Goldberg, The Retina in Systemic Disease: A Color Manual of Ophthalmoscopy. New York, NY: Thieme, 2009.

Authors' photographs and biographies not available at the time of publication. 